\title{
Semantic Completeness of First-Order Theories in Constructive Reverse Mathematics
}

\author{
Christian Espíndola
}

\begin{abstract}
We introduce a general notion of semantic structure for first-order theories, covering a variety of constructions such as Tarski and Kripke semantics, and prove that, over Zermelo-Fraenkel set theory (ZF), the completeness of such semantics is equivalent to the Boolean prime ideal theorem (BPI). Using a result of McCarty (2008), we conclude that the completeness of Kripke semantics is equivalent, over intuitionistic Zermelo-Fraenkel set theory (IZF), to the Law of Excluded Middle plus BPI. Along the way, we also prove the equivalence, over ZF, between BPI and the completeness theorem for Kripke semantics for both first-order and propositional theories.
\end{abstract}

In Henkin [8], the equivalence between the compactness theorem for classical theories and the Boolean prime ideal theorem (BPI) is discussed (see, also Jech [9]). As a consequence, the completeness for such theories is also equivalent to BPI, while, on the other hand, the soundness theorem can be easily proved in Zermelo-Fraenkel set theory ZF by induction on the complexity of the formulas. As we shall see in this note, the fact that the completeness theorem supposes an unavoidable use of a choice principle is not only a feature of classical semantics but also of intuitionistic semantics, and in fact of any semantic structure that is sound and satisfies some reasonable property that we will consider below. This condition puts reasonable restrictions on the interpretations of the connectives, but as we shall see, also conveys to such semantics an intrinsic choice appeal.

That completeness theorems for intuitionistic theories imply nonconstructive principles has been known since 1957, when Gödel derived Markov's principle from the assumption of completeness (see this and related ideas in Kreisel [11], McCarty [13], [14], Carter [5], [6]). Our result will focus on the completeness for the general

Received July 10, 2013; accepted February 15, 2014

First published online February 9, 2016

2010 Mathematics Subject Classification: Primary 03F50, 03E35; Secondary 03E25

Keywords: completeness, constructive reverse mathematics, Kripke semantics, algebraic semantics

(C) 2016 by University of Notre Dame 10.1215/00294527-3470433 


\title{
Semantic Completeness of First-Order Theories in Constructive Reverse Mathematics
}

\author{
Christian Espíndola
}

\begin{abstract}
We introduce a general notion of semantic structure for first-order theories, covering a variety of constructions such as Tarski and Kripke semantics, and prove that, over Zermelo-Fraenkel set theory (ZF), the completeness of such semantics is equivalent to the Boolean prime ideal theorem (BPI). Using a result of McCarty (2008), we conclude that the completeness of Kripke semantics is equivalent, over intuitionistic Zermelo-Fraenkel set theory (IZF), to the Law of Excluded Middle plus BPI. Along the way, we also prove the equivalence, over ZF, between BPI and the completeness theorem for Kripke semantics for both first-order and propositional theories.
\end{abstract}

In Henkin [8], the equivalence between the compactness theorem for classical theories and the Boolean prime ideal theorem (BPI) is discussed (see, also Jech [9]). As a consequence, the completeness for such theories is also equivalent to BPI, while, on the other hand, the soundness theorem can be easily proved in Zermelo-Fraenkel set theory ZF by induction on the complexity of the formulas. As we shall see in this note, the fact that the completeness theorem supposes an unavoidable use of a choice principle is not only a feature of classical semantics but also of intuitionistic semantics, and in fact of any semantic structure that is sound and satisfies some reasonable property that we will consider below. This condition puts reasonable restrictions on the interpretations of the connectives, but as we shall see, also conveys to such semantics an intrinsic choice appeal.

That completeness theorems for intuitionistic theories imply nonconstructive principles has been known since 1957, when Gödel derived Markov's principle from the assumption of completeness (see this and related ideas in Kreisel [11], McCarty [13], [14], Carter [5], [6]). Our result will focus on the completeness for the general

Received July 10, 2013; accepted February 15, 2014

First published online February 9, 2016

2010 Mathematics Subject Classification: Primary 03F50, 03E35; Secondary 03E25

Keywords: completeness, constructive reverse mathematics, Kripke semantics, algebraic semantics

(C) 2016 by University of Notre Dame 10.1215/00294527-3470433 


\title{
Semantic Completeness of First-Order Theories in Constructive Reverse Mathematics
}

\author{
Christian Espíndola
}

\begin{abstract}
We introduce a general notion of semantic structure for first-order theories, covering a variety of constructions such as Tarski and Kripke semantics, and prove that, over Zermelo-Fraenkel set theory (ZF), the completeness of such semantics is equivalent to the Boolean prime ideal theorem (BPI). Using a result of McCarty (2008), we conclude that the completeness of Kripke semantics is equivalent, over intuitionistic Zermelo-Fraenkel set theory (IZF), to the Law of Excluded Middle plus BPI. Along the way, we also prove the equivalence, over ZF, between BPI and the completeness theorem for Kripke semantics for both first-order and propositional theories.
\end{abstract}

In Henkin [8], the equivalence between the compactness theorem for classical theories and the Boolean prime ideal theorem (BPI) is discussed (see, also Jech [9]). As a consequence, the completeness for such theories is also equivalent to BPI, while, on the other hand, the soundness theorem can be easily proved in Zermelo-Fraenkel set theory ZF by induction on the complexity of the formulas. As we shall see in this note, the fact that the completeness theorem supposes an unavoidable use of a choice principle is not only a feature of classical semantics but also of intuitionistic semantics, and in fact of any semantic structure that is sound and satisfies some reasonable property that we will consider below. This condition puts reasonable restrictions on the interpretations of the connectives, but as we shall see, also conveys to such semantics an intrinsic choice appeal.

That completeness theorems for intuitionistic theories imply nonconstructive principles has been known since 1957, when Gödel derived Markov's principle from the assumption of completeness (see this and related ideas in Kreisel [11], McCarty [13], [14], Carter [5], [6]). Our result will focus on the completeness for the general

Received July 10, 2013; accepted February 15, 2014

First published online February 9, 2016

2010 Mathematics Subject Classification: Primary 03F50, 03E35; Secondary 03E25

Keywords: completeness, constructive reverse mathematics, Kripke semantics, algebraic semantics

(C) 2016 by University of Notre Dame 10.1215/00294527-3470433 


\title{
Semantic Completeness of First-Order Theories in Constructive Reverse Mathematics
}

\author{
Christian Espíndola
}

\begin{abstract}
We introduce a general notion of semantic structure for first-order theories, covering a variety of constructions such as Tarski and Kripke semantics, and prove that, over Zermelo-Fraenkel set theory (ZF), the completeness of such semantics is equivalent to the Boolean prime ideal theorem (BPI). Using a result of McCarty (2008), we conclude that the completeness of Kripke semantics is equivalent, over intuitionistic Zermelo-Fraenkel set theory (IZF), to the Law of Excluded Middle plus BPI. Along the way, we also prove the equivalence, over ZF, between BPI and the completeness theorem for Kripke semantics for both first-order and propositional theories.
\end{abstract}

In Henkin [8], the equivalence between the compactness theorem for classical theories and the Boolean prime ideal theorem (BPI) is discussed (see, also Jech [9]). As a consequence, the completeness for such theories is also equivalent to BPI, while, on the other hand, the soundness theorem can be easily proved in Zermelo-Fraenkel set theory ZF by induction on the complexity of the formulas. As we shall see in this note, the fact that the completeness theorem supposes an unavoidable use of a choice principle is not only a feature of classical semantics but also of intuitionistic semantics, and in fact of any semantic structure that is sound and satisfies some reasonable property that we will consider below. This condition puts reasonable restrictions on the interpretations of the connectives, but as we shall see, also conveys to such semantics an intrinsic choice appeal.

That completeness theorems for intuitionistic theories imply nonconstructive principles has been known since 1957, when Gödel derived Markov's principle from the assumption of completeness (see this and related ideas in Kreisel [11], McCarty [13], [14], Carter [5], [6]). Our result will focus on the completeness for the general

Received July 10, 2013; accepted February 15, 2014

First published online February 9, 2016

2010 Mathematics Subject Classification: Primary 03F50, 03E35; Secondary 03E25

Keywords: completeness, constructive reverse mathematics, Kripke semantics, algebraic semantics

(C) 2016 by University of Notre Dame 10.1215/00294527-3470433 


\title{
Semantic Completeness of First-Order Theories in Constructive Reverse Mathematics
}

\author{
Christian Espíndola
}

\begin{abstract}
We introduce a general notion of semantic structure for first-order theories, covering a variety of constructions such as Tarski and Kripke semantics, and prove that, over Zermelo-Fraenkel set theory (ZF), the completeness of such semantics is equivalent to the Boolean prime ideal theorem (BPI). Using a result of McCarty (2008), we conclude that the completeness of Kripke semantics is equivalent, over intuitionistic Zermelo-Fraenkel set theory (IZF), to the Law of Excluded Middle plus BPI. Along the way, we also prove the equivalence, over ZF, between BPI and the completeness theorem for Kripke semantics for both first-order and propositional theories.
\end{abstract}

In Henkin [8], the equivalence between the compactness theorem for classical theories and the Boolean prime ideal theorem (BPI) is discussed (see, also Jech [9]). As a consequence, the completeness for such theories is also equivalent to BPI, while, on the other hand, the soundness theorem can be easily proved in Zermelo-Fraenkel set theory ZF by induction on the complexity of the formulas. As we shall see in this note, the fact that the completeness theorem supposes an unavoidable use of a choice principle is not only a feature of classical semantics but also of intuitionistic semantics, and in fact of any semantic structure that is sound and satisfies some reasonable property that we will consider below. This condition puts reasonable restrictions on the interpretations of the connectives, but as we shall see, also conveys to such semantics an intrinsic choice appeal.

That completeness theorems for intuitionistic theories imply nonconstructive principles has been known since 1957, when Gödel derived Markov's principle from the assumption of completeness (see this and related ideas in Kreisel [11], McCarty [13], [14], Carter [5], [6]). Our result will focus on the completeness for the general

Received July 10, 2013; accepted February 15, 2014

First published online February 9, 2016

2010 Mathematics Subject Classification: Primary 03F50, 03E35; Secondary 03E25

Keywords: completeness, constructive reverse mathematics, Kripke semantics, algebraic semantics

(C) 2016 by University of Notre Dame 10.1215/00294527-3470433 


\title{
Semantic Completeness of First-Order Theories in Constructive Reverse Mathematics
}

\author{
Christian Espíndola
}

\begin{abstract}
We introduce a general notion of semantic structure for first-order theories, covering a variety of constructions such as Tarski and Kripke semantics, and prove that, over Zermelo-Fraenkel set theory (ZF), the completeness of such semantics is equivalent to the Boolean prime ideal theorem (BPI). Using a result of McCarty (2008), we conclude that the completeness of Kripke semantics is equivalent, over intuitionistic Zermelo-Fraenkel set theory (IZF), to the Law of Excluded Middle plus BPI. Along the way, we also prove the equivalence, over ZF, between BPI and the completeness theorem for Kripke semantics for both first-order and propositional theories.
\end{abstract}

In Henkin [8], the equivalence between the compactness theorem for classical theories and the Boolean prime ideal theorem (BPI) is discussed (see, also Jech [9]). As a consequence, the completeness for such theories is also equivalent to BPI, while, on the other hand, the soundness theorem can be easily proved in Zermelo-Fraenkel set theory ZF by induction on the complexity of the formulas. As we shall see in this note, the fact that the completeness theorem supposes an unavoidable use of a choice principle is not only a feature of classical semantics but also of intuitionistic semantics, and in fact of any semantic structure that is sound and satisfies some reasonable property that we will consider below. This condition puts reasonable restrictions on the interpretations of the connectives, but as we shall see, also conveys to such semantics an intrinsic choice appeal.

That completeness theorems for intuitionistic theories imply nonconstructive principles has been known since 1957, when Gödel derived Markov's principle from the assumption of completeness (see this and related ideas in Kreisel [11], McCarty [13], [14], Carter [5], [6]). Our result will focus on the completeness for the general

Received July 10, 2013; accepted February 15, 2014

First published online February 9, 2016

2010 Mathematics Subject Classification: Primary 03F50, 03E35; Secondary 03E25

Keywords: completeness, constructive reverse mathematics, Kripke semantics, algebraic semantics

(C) 2016 by University of Notre Dame 10.1215/00294527-3470433 